\title{
Fear flows as efforts to ease blood shortage continue in vein
}

Emerging infections such as West Nile virus, mad cow disease and foot-and-mouth disease are shrinking the global blood supply, which was already threatened by the AIDS epidemic. The dwindling reserves, say experts, could spell doom for hospitals worldwide.

US blood banks have enough blood to meet demands for roughly two or three days. But a five-to-seven day margin is optimal, says Roger Dodd, president of the American Association of Blood Banks. Worldwide, the picture is equally grim: there are about 75 million blood collections every year, but the majority of the world's population has access to only $40 \%$ of the available blood. The reserves are also falling because of a shifting demographic, adds Dodd. "People seem less and less inclined to give blood," he says.

For years, scientists have been trying to create viable blood substitutes, but that goal has been elusive. Two US products are now close to reaching the clinic: Hemopure, made by Cambridge-based Biopure, and PolyHeme, developed by Northfield Laboratories of Illinois. Neither can replace blood entirelymost substitutes focus primarily on transporting oxygen - but will at least serve as a "bridge" in emergency situations, their makers say.

Hemopure, made from purified bovine blood, is farthest along in the regulatory process. Already approved in South Africa, it delivers oxygen three times more efficiently than red blood cells and can be administered

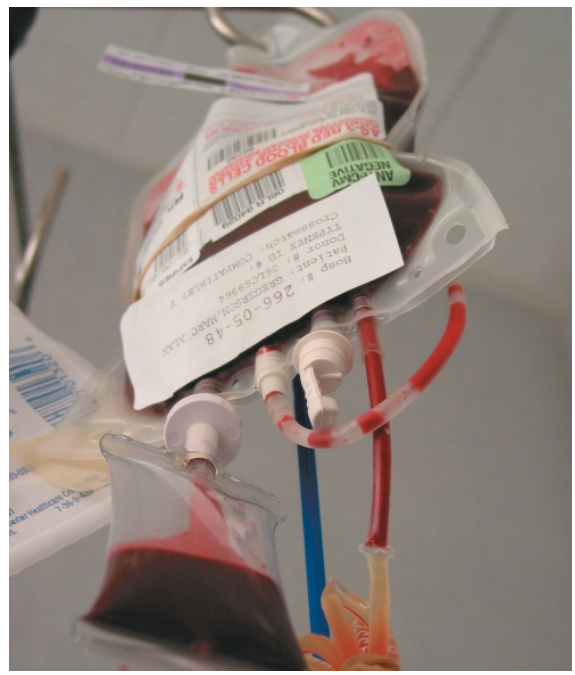

Blood lines: Screening for infectious agents is bleeding reserves dry, experts warn

regardless of blood type, says Biopure spokesman Douglas Sayles. Hemopure also has a three-year shelf life-much longer than the 42-day life span of red blood cells.

Once inside a patient however, Hemopure lasts only for 12-24 hours, whereas transfused red blood cells can live for up to 50 days. Scientists remain skeptical about blood substitutes for that reason, calling them a short-term solution useful only for specific indications such as military operations and heavy trauma episodes, where physicians need blood quickly.
Creating substitutes for blood is challenging because of the multitasking nature of the viscous life force: blood carries oxygen, nutrients and waste products, and helps repair injuries. Most substitutes are derived from oxygen-carrying perfluorocarbons or natural hemoglobin. But Fluosol, the only blood substitute ever approved by the US Food and Drug Administration (FDA), was taken off the market because patients given Fluosol breathed pure oxygen - which can be toxic.

Hemoglobin-based substitutes stem from outdated human blood, cow blood or recombinant hemoglobin produced by bacterial cells. Recently, the common marine worm was suggested as another promising alternative. But hemoglobin-based substitutes also carry the risk of toxic side effects, such as inflammation and oxygen stress.

Some researchers are trying to grow blood vessels in a test tube. A Harvard University research team also recently reported that adding uridine diphosphate-galactose to blood platelet cells increases their shelf life from 5 days to 12 (Science 301, 1531-1534; 2003)

The real test for any substitute, says Tom Moore, chief executive officer of Biopure, is getting FDA approval. "It is a challenge to run a clinical trial against a natural product," Moore says. "Blood has never really been characterized, so the standards we are putting ourselves against are murky."

Paroma Basu, New York

\section{European researchers up in arms over new clinical trials edict}

European researchers are appealing to their national governments to reject a sweeping new directive on clinical trials. The edict, which must first be incorporated into the law of each European Union (EU) country, is expected to take effect on 1 May 2004.

The directive is intended to standardize and simplify the rules governing clinical trials. But researchers fear that layers of bureaucracy will only add to the regulatory hurdles and cost of clinical trials, without improving patient safety or trial quality (Nat. Med. 7, 1264; 2001)

"There is a real risk that European public health policy makers and national authorities may marginalize key areas of biomedical and health research by developing [these] policies," says Françoise Meunier, director general of the Brussels-based European Organization for Research and Treatment of Cancer.

The measure proposes one model for all clinical trials, including those sponsored by acade- mia, industry, charities and government bodies. The model closely resembles one currently used by the pharmaceutical industry and makes researchers responsible for sponsorship, manufacturing authorization and good manufacturing practices (GMP), as well as a huge amount of paperwork on trial authorization and registration, data verification and pharmacovigilance.

Unlike the drug industry, academic centers rarely have the expertise required to build and run GMP-compliant facilities, and some observers already foresee competition with industry for suitable staff. Critics of the directive argue that it was primarily designed with industry in mind, and that the reality of academic trials was ignored.

"The EU rules must move into line with reality and feasibility," says Jaap Verweij, a researcher at the Erasmus Medical Center in Rotterdam. "[Otherwise], academic clinical research will come to a standstill and global collaboration will cease."

Under the directive, nationwide trials will be subject to review by a single ethical committee. But assessing the suitability of staff and locations at distant sites is virtually impossible and is likely to be delegated to the host institutions, notes Simon Thomas, a clinical researcher at the University of Newcastle upon Tyne. As a result, Thomas says, "independent review of locality issues for multicenter studies will be lost."

Another issue is cost. According to a study by Cancer Research UK, increased bureaucracy from the legislation will double the paperwork and quadruple the cost of publicly funded clinical trials. As a result, warns Peter Selby, director of the organization's clinical center at St. James University in Leeds, there will be fewer trials available to patients, and important clinical questions will remain unanswered.

Xavier Bosch, Barcelona 